\title{
Novel cutting inserts with multi-channel irrigation at the chip- tool interface: modelling, design and experiments
}

\author{
a Machining and Condition Monitoring Group, Faculty of Engineering, University of Nottingham, UK \\ ${ }^{b}$ Faculty of Engineering, University of Nottingham Ningbo, China \\ c Seco Tools AB, R\&D Material and Technology Development, Fagersta, Sweden \\ ${ }^{d}$ GKN Aerospace Engine Systems AB, Sweden
}

Zhirong Liao, Dongdong Xua, Dragos Axintea,b,*,(1), Rachid M’Saoubic(1), Jimmy Thelinc, Anders Wretland

The friction at chip-tool interface can considerably affect the chip formation and consumed energy during cutting of superalloys. However, it is difficult to deliver the lubricant to the chip-tool interface to reduce the friction effect. Thus, this paper proposed a novel solution of insert design by locating macrochannels on the rake face which connect with the micro-channels for irrigating the coolant into the chip-tool interface, while considering the cooling and lubricating efficiency. A significant reduction of tool wear, cutting force and specific cutting energy has been demonstrated, while an improved chip fragmentation as well as microstructure has also been achieved.

Cutting tool; cooling; chip-tool interface

\section{Introduction}

With more advanced materials, e.g. superalloys, applied in various high-value industries, the cutting tool performance is facing critical challenges due to the complex properties of those materials such as high strength at high temperature, which thereby lead to severe thermomechanical loads and result in accelerated tool wear [1-4]. High pressure coolant is of advantage to reduce the cutting temperature and enhance chip control in superalloys machining to enable adequate surface integrity and cutting tool performance [5-7]. However, the conventional solutions (Fig. 1a) for high-pressure cutting fluid delivery rely on "macro-nozzles" (i.e. dimensions comparable with those of the cutting tools) positioned outside the chip-tool interface that miss to deliver the coolant at the critical tool-chip/workpiece contact zones efficiently $[6,8]$. This is because, the high contact stresses developed at the chip-tool interface restrain the penetration of the coolant into the cutting area [9]. Thus, the cooling and lubricating effects of the fluid are limited within the cutting zone and the highpressure coolant mainly results in better curling and fragmenting the chips but with limited increase of tool life $[9,10]$.

On the other hand, benefiting from the development of surface engineering technologies, e.g. laser ablation, there are increasing researches on the surface texturing of tools to reduce the cutting friction [11]. This gives the advantages to reduce the contact area between tool rake surface and the chips thus, lowering the frictional as well as the cutting forces $[12,13]$. However, this does not increase the cooling effect as the coolant cannot be delivered to the tool-chip contact area, even with high pressure coolant [13].

To address these challenges, this paper reports on a novel solution, i.e. network of micro-channels coupled to macrochannels, wherein high pressure fluid is supplied at the very end of the cutting edge, i.e. chip-tool interface, which enables the reduction of friction forces. This is a novel concept of applying "lubrication cushions" at the most thermally and mechanically loaded locations of the cutting tool through a 3D network of microchannels that infiltrates cutting fluid into the cutting edge body.

\section{Novel concept of tool design}

As shown in Fig. 1b, in the proposed solution the cutting fluid is supplied through a set of macro discharge channels which are designed closely to the cutting edge and terminate on the rake face. To allow the spatial application of high pressure fluid into "real chip-tool interface", an innovative network of preferentially oriented micro-channels are then engineered on the rake face that are connected with these macro-channels, which infiltrate the fluid to act as a "cushion" for the chip sliding thereon (see Fig. 1c), thereby providing positive effects by reducing the local friction and temperature.

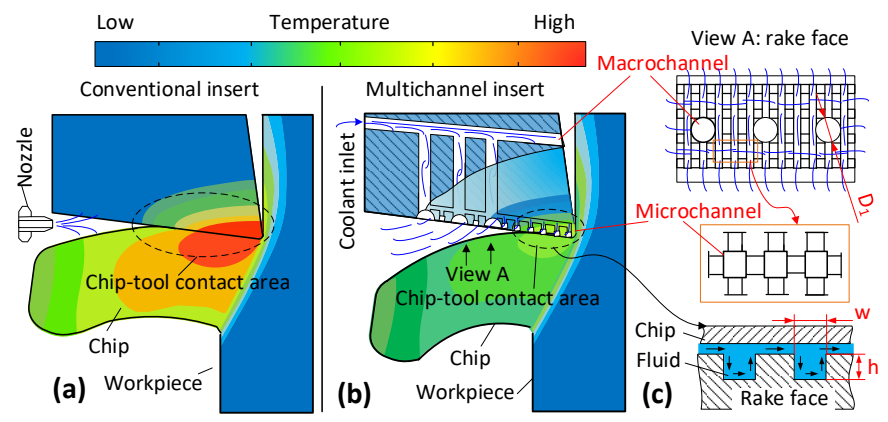

Fig. 1. The concept of proposed multichannel irrigation cutting insert

\subsection{Design on the macro coolant channels}

Since turbulent flow with high Reynolds number ( $>4000$ ) would increase the heat transfer rate, when designing the macrochannels (Fig. 1b), it is necessary to achieve turbulent motion of the coolant flow by applying following condition [13]:

$$
R e=V_{1} D_{1} / v>4000
$$

where $D_{1}$ is the diameter of macro channels and $V_{1}$ is the fluid speed. Considering the viscosity $v$ of cutting fluid is in the order of $10^{-6} \mathrm{~m}^{2} / \mathrm{s}$ [13], the design criterion can be: $V_{1} D_{1}>0.004 \mathrm{~m}^{2} / \mathrm{s}$.

Based on Bernoulli's law and the coolant flow condition in cutting insert, the fluid speed $\left(\mathrm{V}_{1}\right)$ can be determined as

$$
V_{1}=\sqrt{2 P_{1} / \rho_{f}}
$$

where $\rho_{f}$ is the coolant density and $P_{1}$ the coolant pumping pressure. Considering the coolant pressure can be varied for different applications, the lower limit pressure $\left(P_{1}=10 \mathrm{bar}\right)$ is applied for the design calculation to ensure that the proposed multi-channel irrigation insert will provide the required performance. Hence, with conventional water based cutting fluid where $\rho_{f}=1000 \mathrm{~kg} / \mathrm{m}^{3}$, the fluid speed at coolant discharge can determined as $V_{1} \approx 45 \mathrm{~m} / \mathrm{s}$.

To allow the efficient cooling during the machining process, a set of macro coolant discharge channels were designed on the rake and clearance face, as shown in Fig. 2, where the coolant is supplied through an internal passage. Assuming the fluid speeds at all 
discharges on the insert are the same, then based on above mentioned design criterion the diameter of coolant discharges can be approximated as $D_{1}>88 \mu \mathrm{m}$.

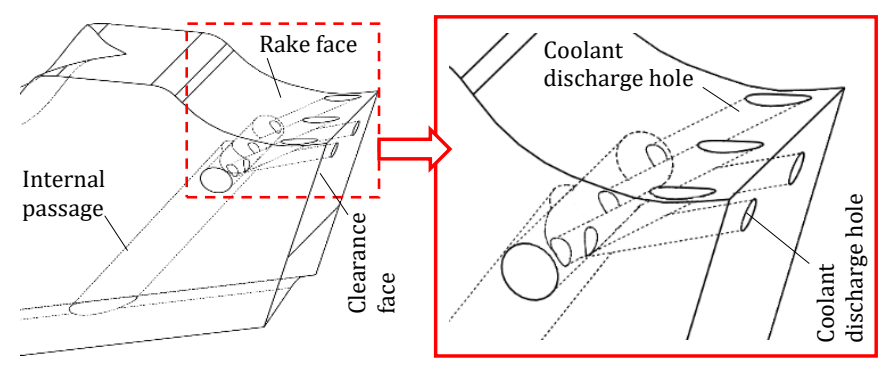

Fig. 2. Designed macro-channels on the insert rake and clearance faces

Considering the EDM manufacturing capability, the diameter of the macro channels was selected as $D_{1}=300 \mu \mathrm{m}$. Finite element (FE) analysis (ABAQUS) of the insert (Fig. 3a) on machining of Inconel 718 at cutting speed of $30 \mathrm{~m} / \mathrm{min}$ and feed rate of $0.2 \mathrm{~mm} / \mathrm{rev}$ shows that the maximum machining induced stress $(2.1 \mathrm{GPa})$ in the "weakened" tool structure that contains these macro-channels is far below the tool (tungsten carbide) strength (5GPa). Hence, the suggested design solution can be applied for real cutting without the risk of cutting edge fracture. To validate the fluid speed, computational fluid dynamics (CFD) simulation (FLUENT) has also been employed (Fig. 3 b), whereby a maximum flow speed of 51 and $128 \mathrm{~m} / \mathrm{s}$ was achieved under the pressure of 10 and $70 \mathrm{bar}$ respectively, close to the analytical results of 45 and $119 \mathrm{~m} / \mathrm{s}$.
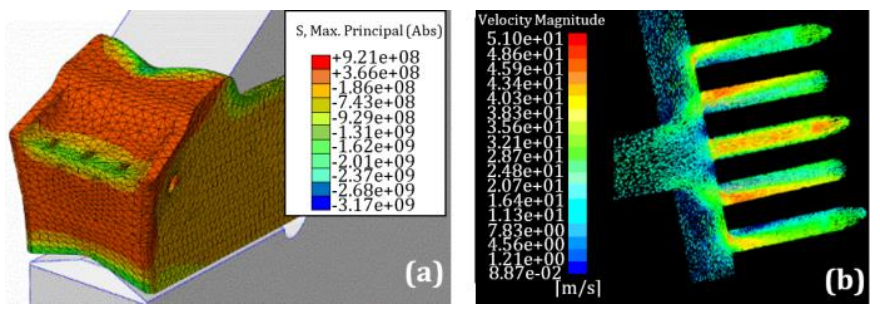

Fig. 3. Simulation of the macro-channel insert design: (a) FE for structure strength validation and (b) CFD for macro-channel fluid speed validation.

\subsection{Design of the micro coolant channels}

While a set of micro-channels are engineered on the tool rake face to connect the macro-channels to the cutting edge (Fig. 1), and considering that the static pressure in the microchannel is negligible, the fluid speed $V_{2}$ in this area can be evaluated as $V_{2}=V_{1}$.

Hence to approach the turbulent condition, the equivalent diameter $D_{2}$ of the microchannels should meet the condition of $D_{2}>88 \mu \mathrm{m}$. However, when fabricating the micro-channels (i.e. small pockets) on the insert surface using pulsed laser texturing, the cross section can be approximated with a rectangle which characteristics (w - width and $h$ - depth) can be derived as follows:

$$
D_{2}=4 \frac{w h}{2(w+h)}>88 \mu m
$$

Nevertheless, during the cutting process the adhesive friction force $\left(F_{f}\right)$ between the tool and the sliding chip can be calculated as [7]:

$$
F_{f}=A_{w} \tau_{c}
$$

where $\tau_{c}$ and $A_{w}$ are the shear strength and contact area between the tool and chip respectively. Thus, a larger channel width leads to a lower tool-chip contact area, hence a reduced adhesive friction. With also considering the manufacturability limitation of the pulsed laser, the width $w$ and depth $h$ are then selected as $100 \mu \mathrm{m}$.

\subsection{Tool fabrication}

For prototype a tungsten carbide cutting insert from Seco Tools $(150.10-4 \mathrm{~N}-12)$ with the rake angle $10^{\circ}$, clearance angle $15^{\circ}, 4 \mathrm{~mm}$ width was selected to be engineered with the proposed concept. According to the above calculation the dimension of the macro and micro-channels can be designed as listed in Table 1. The macrochannels were drilled by EDM while the micro-channel network was generated by a picosecond pulsed laser at a distance of $200 \mu \mathrm{m}$ from the cutting edge. Fig. 4 shows the 2D (Fig. 4a: scanning electron microscopy-SEM) and 3D (Fig. 4b: Optical-Alicona) measurement of the insert prototype.

Table 1 The tool fabrication parameters

\begin{tabular}{lc|ccc}
\hline \multicolumn{2}{l|}{ Macro-channel $(\mu \mathrm{m})$} & \multicolumn{3}{c}{ Micro-channel $(\mu \mathrm{m})$} \\
\hline Inlet & Outlet & Depth & Width & Pitch \\
700 & 300 & 100 & 100 & 150 \\
\hline
\end{tabular}
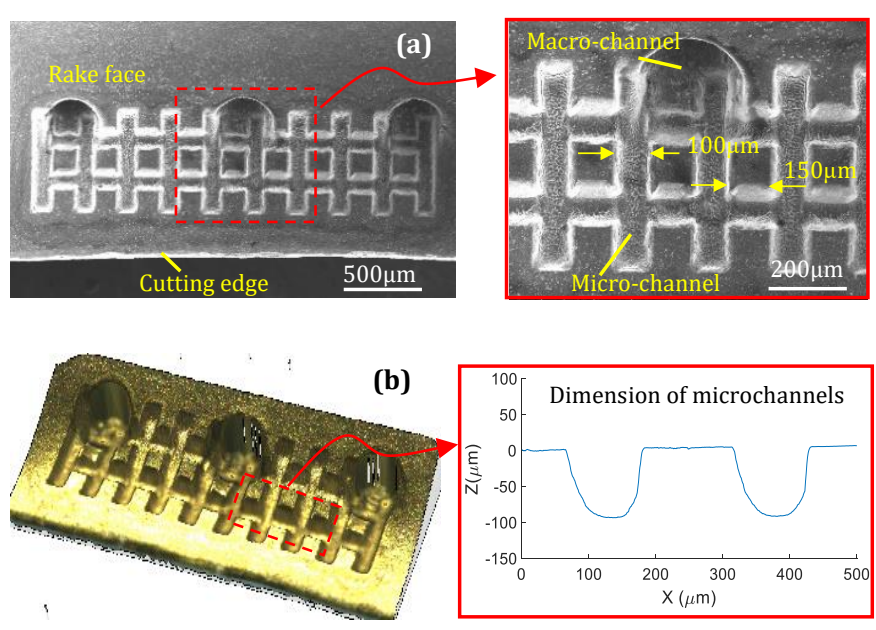

Fig. 4. Details of tool macro/micro channels: (a) SEM and (b) 3D profile

\section{Experimental details}

To validate the ability of the proposed multi-channel insert against conventional one, orthogonal cutting tests were carried out on a CNC lathe with Inconel 718 as workpiece material. Two different cutting speeds ( $\mathrm{vc}=30$ and $60 \mathrm{~m} / \mathrm{min}$ ) with the feed rate of $0.2 \mathrm{~mm} / \mathrm{rev}$ were chosen while the cutting width was set as $2 \mathrm{~mm}$ to allow the plain strain condition, as shown in Fig. 5. A fixed length of $20 \mathrm{~m}$ was cut for each test with 70 bar fluid pressure supplied internally for multi-channel insert and externally for conventional insert.
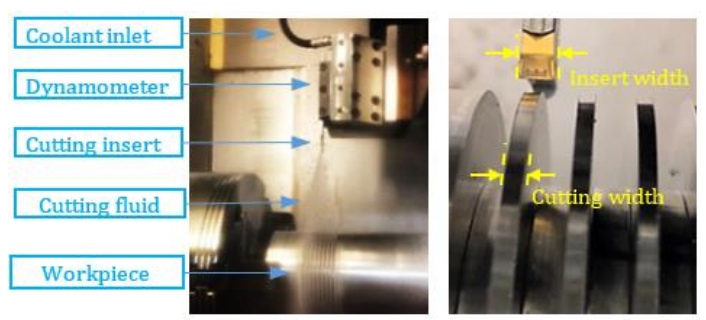

Fig. 5. Orthogonal cutting test with the multi-channel insert

\section{Results and discussion}

\subsection{Chip-tool contact area}

Due to the high normal and shear stress from the chip, severe friction occurs in cutting zone (Fig. 6a), which generates significant 
sticking and sliding wear (Fig. $6 \mathrm{~b}$ and c) on the rake face. More interestingly, although identical wear widths $(2 \mathrm{~mm})$ are observed on these two inserts due to the same cutting width, there is a clear difference in the sticking length wherein the proposed insert is much shorter (max. $0.45 \mathrm{~mm}$ ) than the conventional insert (max. $0.9 \mathrm{~mm}$ ), with a reduction of $\sim 50 \%$. This indicates a better cutting fluid infiltration at the chip-tool interface of the proposed insert, resulting in an enhanced lubricating and cooling effect hence lower tool wear.

More evidently, apart from the difference of the chip-tool contact length, the wear pattern also illustrates the disparity between the two inserts, wherein a periodically decrease of wear area at the locations of macro-channel entry appears due to a better coolant delivery from the coolant discharge to the coupled micro-channels. In contrast, a larger rectangular wear pattern is observed in the normal insert, indicating a more uniform and severe wear effect.
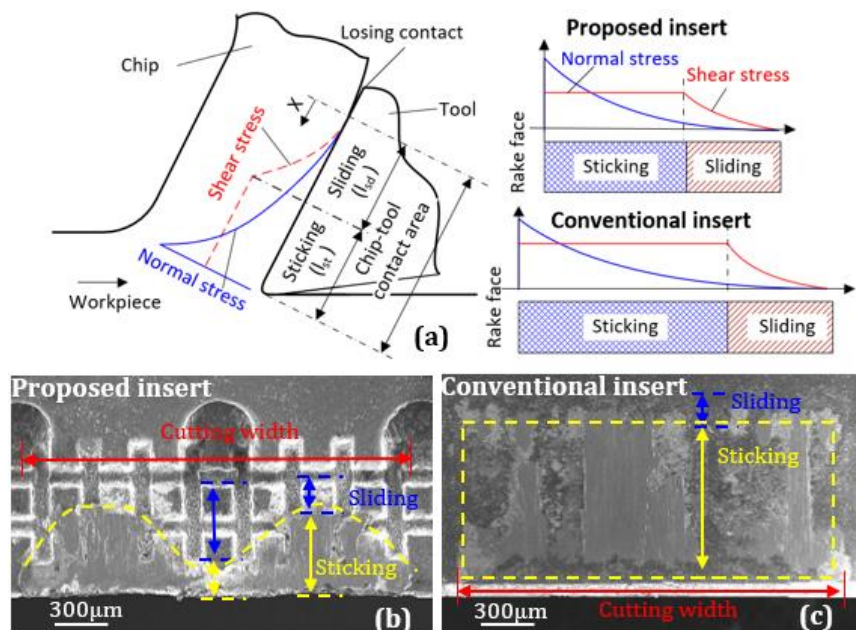

Fig. 6. Friction effect on the chip-tool contact area: (a) schematic illustration [7], (b) proposed and (c) conventional insert

\subsection{Chip morphology and friction at chip-tool interface}

An improvement of the chip fragmentation can also be observed from chip geometry (Fig. 7) where a long and continuous spiral chip (Fig. $7 \mathrm{~b}$ ) generated from conventional insert at $\mathrm{vc}=30 \mathrm{~m} / \mathrm{min}$ while the proposed insert can break the chip more effectively (Fig. $7 a)$ at the same cutting speed. This is due to the effective fluid jet delivery from the well-located macro-channels which leads to a reduction of the chip-tool contact length while also tends to lift up and break the chip. It is also interesting to observe that when increasing the cutting speed to $60 \mathrm{~m} / \mathrm{min}$, both the proposed and conventional insert could break the chip effectively due to the increased strain rate and cutting temperature.
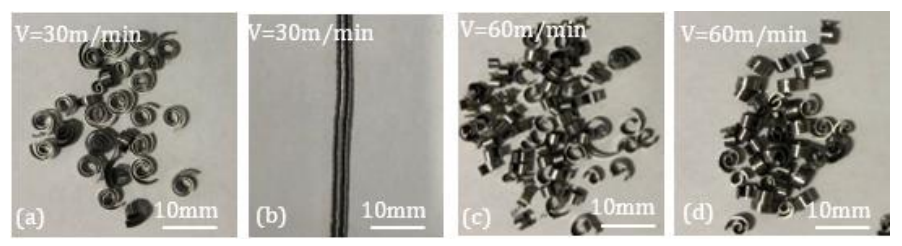

Fig. 7. Different chip fragmentation effect with: (a) and (c) proposed insert, (b) and (d) conventional insert

Nevertheless, the chip morphology from SEM images (Fig. 8) shows that the conventional insert generated more severe serrated chip with larger thickness compared with proposed insert. This considerable crushing and deformation can be accounted by the more significant chip compression phenomenon from adiabatic shearing as well as the friction effect of the conventional insert during the chip formation process.
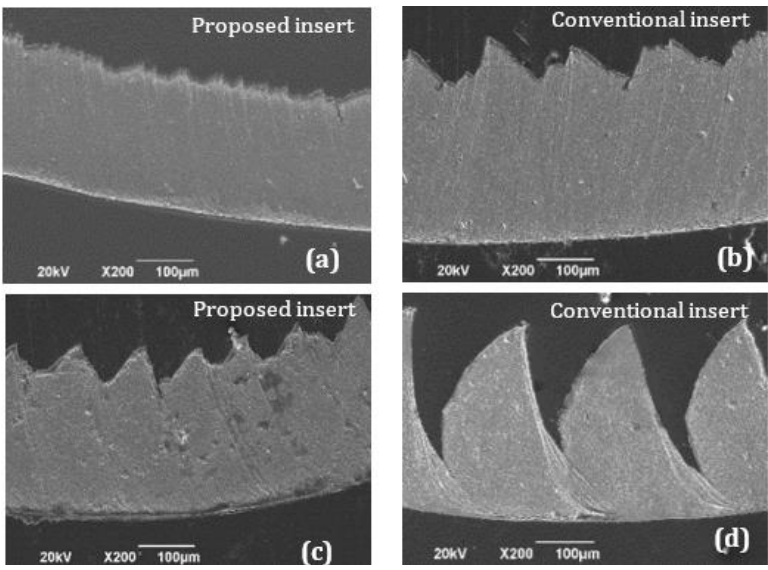

Fig. 8. Chip morphology: (a) and (b) V=30m/min, (c) and (d) V=60m/min

Since the chip segmentation is critically related to the localised shear strain and friction effect, to quantify this phenomenon the degree of chip segmentation G can be calculated as $\left(H_{\max }\right.$ and $H_{\min }$ are the maximum and minimum chip thickness respectively).

$$
G=\left(H_{\max }-H_{\min }\right) / H_{\max }
$$

Moreover, the friction effect at the chip-tool contact area can be also quantified from the friction coefficient, which is approximated from the chip morphology based on metal cutting principles [7]. The thickness of the segmented chip is approximated as $\left(H_{\max }+H_{\min }\right) / 2$ when calculating the shear plane angle.
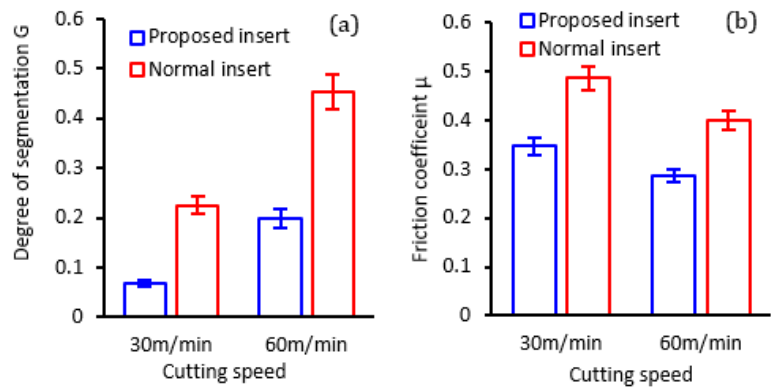

Fig. 9. Details of (a) degree of segmentation and (b) friction coefficient

As shown in Fig. 9(a), the degree of segmentation from conventional insert is twice over the proposed insert, indicating a severer adiabatic shearing effect. This rise of shearing effect can also be supported from the friction coefficient observation, as shown in Fig. 9(b), where a 30\% decrease from proposed insert can be seen compared with the conventional insert.

4.3. Cutting force and energy

As a prominent indicator of the cooling and lubricating effect the cutting force has also been compared (Fig. 10), where a significant decrease of the average feed force (ca. $29 \%$ and $27 \%$ for vc $=30$ and $60 \mathrm{~m} / \mathrm{min}$ respectively) and cutting force (ca. $19 \%$ and $20 \%$ correspondingly) has been achieved for the proposed insert. 

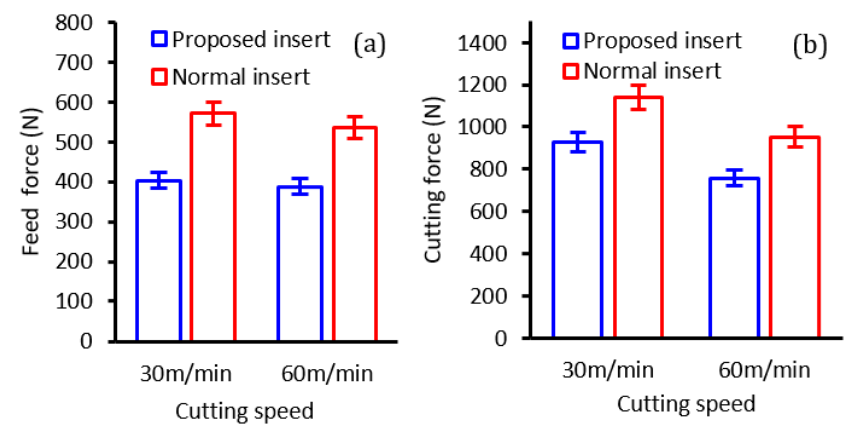

Fig. 10. Comparison of (a) feed force and (b) cutting force

This improvement of cooling and lubricating effect can also be illustrated from specific shear and friction energy respectively. As shown in Fig. 11, a significant decrease of shear energy (ca. $25 \%$ and $28 \%$ for $\mathrm{V}=30$ and $60 \mathrm{~m} / \mathrm{min}$ respectively) and friction energy (ca. $13 \%$ and $12 \%$ correspondingly) has been achieved with the proposed insert. This observation indicates that the proposed insert can effectively deliver the coolant to the cutting area to reduce the adiabatic shear effect (shear energy) as well as the friction effect (friction energy) thus to reduce the shearing deformation and the chip-tool contact area.
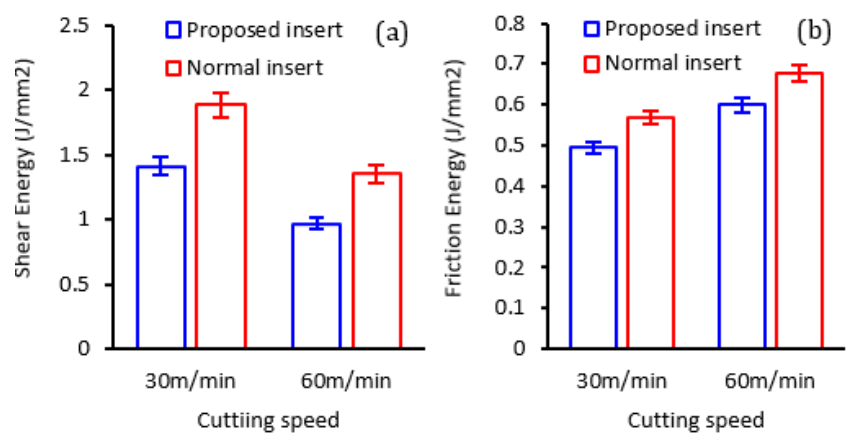

Fig. 11. Comparison of (a) shear and (b) friction energy
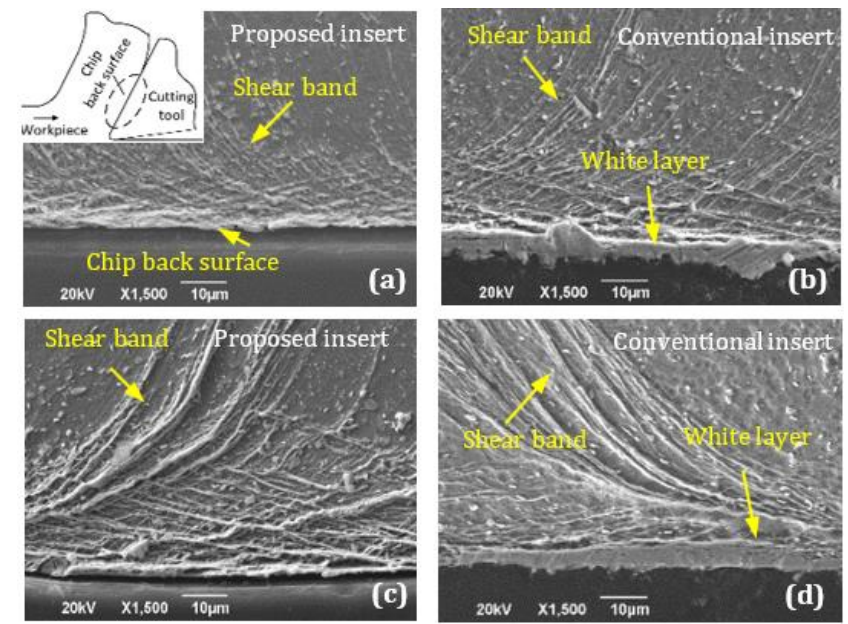

Fig. 12. Microstructure on the cross section of chip back surface: (a) and (b) $\mathrm{V}=30 \mathrm{~m} / \mathrm{min}$, (c) and (d) $\mathrm{V}=60 \mathrm{~m} / \mathrm{min}$

A clear friction effect on the underside of the chip has also been observed where a white layer $(\sim 8 \mu \mathrm{m})$ forms on the chips from conventional insert (Fig. 12b and d) due to high temperature and plastic strain. On the contrary, there is no clear evidence of white layer observed from the chip of proposed insert (Fig. 12a and c), indicating a distinct cooling/lubricating effect between the chip and tool which results in lower normal and shear stress in chiptool interface, as shown in Fig. 6. This, on the other hand, proves the advantage of the proposed insert in terms of more efficient coolant delivery.

\section{Conclusions}

A novel cutting insert has been proposed, on which a set of macro-channels have been designed closely to cutting edge and coupled with a network of micro-channels that infiltrated with the fluid in the tool-chip interface to act as a "cushion" for the chips to slide on. Modelling and experimental work has been conducted to support the design of the proposed concept.

The test results indicate that the proposed insert can enhance its cooling and lubricating performance with multi-channels irrigation at chip-tool interface. As a consequence, the cutting force and specific energy has been reduced significantly (ca. 20\%) benefiting from both the shearing and friction reduction. A reduced chip-tool contact area has been observed, especially on diminishing the sticking wear (ca. 50\%) with a decreased friction coefficient (ca. 30\%). The chip morphology shows an improved fragmentation and evacuation phenomenon of the proposed insert while a reduced adiabatic shearing effect has been achieved due to the reduction of heat. A clear elimination of white layer on the chip underside surface also demonstrates the capability of friction reduction.

These encouraging experimental results underline the potential of the proposed tool design concept for practical machining applications and the need to explore more cost-effective manufacturing routes (e.g. powder metallurgy combined with texture imprinting technology) to upscale the manufacturing of such inserts.

\section{Acknowledgement}

This project has received funding from the Clean Sky 2 Joint Undertaking under European Union's Horizon 2020 research and innovation programme (grant No.754807). The authors thank the support from Nottingham research fellowship programme and H2020-MSCA-COFUND-2014, INNOVATE (grant No. 665468).

\section{References}

[1] Liao Z. et al (2018) On the influence of gamma prime upon machining of advanced nickel based superalloy. CIRP Annals 67(1): 109-112.

[2] Shang Z. et al (2019). On modelling of laser assisted machining: Forward and inverse problems for heat placement control. Int. J. Mach. Tools Manuf., 138: 36-50.

[3] Diaz 0.G. et al (2019) The new challenges of machining Ceramic Matrix Composites (CMCs): review of surface integrity. Int. J. Mach. Tools Manuf., 139: 24-36.

[4] Liao Z. et al (2019) State-of-the-art of surface integrity in machining of metal matrix composites. Int. J. Mach. Tools Manuf., 143: 63-91.

[5] Wertheim R. et al (1992) Influence of high-pressure flushing through the rake face of the cutting tool. CIRP annals 41(1): 101-106.

[6] Ezugwu EO. et al (2004) Effect of high-pressure coolant supply when machining Inconel 718 with coated carbide tools. J. Mater. Process. Technol. 153: 1045-1050.

[7] Shaw C. (2005) Metal Cutting Principles, Vol. 2. Oxford University Press, New York [8] Xu W. and Zhang L. (2019). Heat effect on the material removal in the machining of fibre-reinforced polymer composites. Int. J. Mach. Tools Manuf., 140: 1-11.

[9] Sharma V. et al (2016) Recent advances in turning with textured cutting tools: a review. Journal of Cleaner Production, 137: 701-715.

[10] Tahmasebi E. et al (2019). CFD and experimental analysis of the coolant flow in cryogenic milling. Int. J. Mach. Tools Manuf., 140, 20-33.

[11] Koshy P. et al (2011) Performance of electrical discharge textured cutting tools. CIRP Annals, 60: 153-156.

[12] Enomoto T. et al (2012) Highly wear-resistant cutting tools with textured surfaces in steel cutting. CIRP Annals, 61: 571-574.

[13] Fang Z. et al (2017). Turning of Inconel 718 using inserts with cooling channels under high pressure jet coolant assistance. J. Mater. Process. Technol. 247: 19-28 